\title{
Accuracy Evaluations and Frequency Comparison of NIST-7 and CRL-O1
}

\author{
W. D. Lee, R. E. Drullinger, J. H. Shirley, C. Nelson, D. A. Jennings, \\ L. O. Mullen, F. L. Walls, and T. E. Parker \\ National Institute of Standards and Technology \\ Time and Frequency Division \\ 325 Broadway, Boulder, CO USA \\ and \\ A. Hasegawa, K. Fukuda, N. Kotake, M. Kajita, and T. Morikawa, \\ Communications Research Laboratory \\ 4-2-1, Nukui-Kitamachi, Koganei, Tokyo 184, Japan
}

\begin{abstract}
CRL and NIST have completed the development of CRL-O1, an improved optically pumped primary frequency standard. The results of the first evaluation of the new standard and its comparision with NIST-7, the US primary frequency, standard are presented.
\end{abstract}

\section{INTRODUCTION}

The Communications Research Laboratory (CRL) of Japan and the National Institute of Standards and Technology (NIST) of the US have completed the development and accuracy evaluation of CRL-O1, an optically pumped, thermal-atomic-beam primary frequency standard. Advancements in the beam tube, electronics, and computer software are presented. Both CRL-O1 and NIST-7 were evaluated at NIST over the same time. The fractional-frequency difference between the two standards was $1 \cdot 10^{-15} \pm 6 \cdot 10^{-15}$ $(1 \sigma)$.

\section{Atomic Beam Tube}

The atomic beam tube of CRL-O1 is similar to that of NIST-7 [1]. It has a Ramsey cavity $1.55 \mathrm{~m}$ in length. The atomic-beam diameter is $3 \mathrm{~mm}$. The axial C-field is produced by a solenoid that covers not only the microwave interrogation regions but also the optical state preparation and detection zones.

The laser radiation for optical state preparation and detection is produced by a distributed-Braggreflector (DBR) semiconductor laser. Measurements made with CRL-O1 indicate that this improved laser

Contribution of the U. S. Government, not subject to copyright. system has extremely low sensitivity to seismic and acoustic disturbances.

\section{Digital Frequency Servo}

CRL-O1 and NIST-7 are not operated as clocks. Rather, they are used to periodically measure the frequency of a stable reference oscillator with respect to the atomic resonance with known biases removed. This reference oscillator serves as a transfer standard for conveying frequency information to BIPM. Figure 1 is a block diagram of the digital servo that compares the reference oscillator to the atomic resonance. The digital servo performs slow square-wave frequency modulation of a microwave synthesizer [2] using a computer-controlled, direct-digital synthesizer (DDS). The fluorescence signal from the cesium beam is digitized by an analog-to-digital converter and then demodulated in software, producing a frequency error signal. A software controller then steers the DDS center frequency to force this error signal to 0 . The set of frequency-steering corrections $\Delta f[i]$ is stored by the computer for later calculation of the mean frequency difference between the reference oscillator and the atomic resonance.

\section{Digital Power Servo}

The computer must maintain a constant microwave power throughout a frequency measurement. Several pitfalls associated with the microwave power control have been eliminated with the development of CRLO1: microwave leakage, spurious RF sidebands, and ground loops. Figure 2 is a block diagram of the power attenuator. Since microwave leakage is a po- 


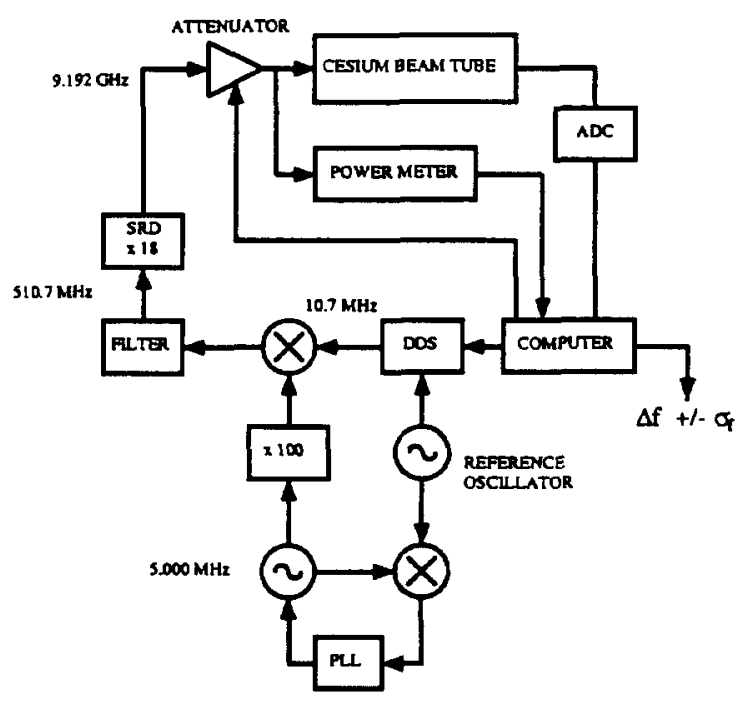

Figure 1: Digital Frequency Servo.

$\mathrm{SRD}=$ step-recovery diode, $\mathrm{DDS}=$ direct digital synthesizer, PLL=phase-locked loop, ADC=analog-todigital converter.

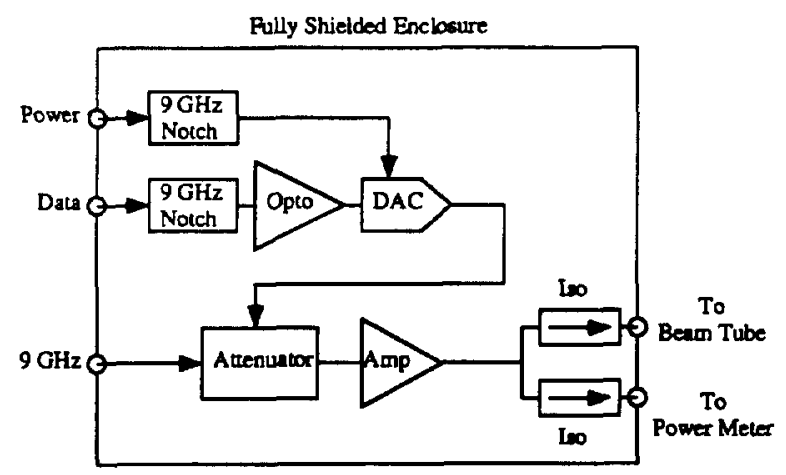

Figure 2: Microwave Power Attenuator.

Opto=digital optical isolator, $\mathrm{DAC}=$ Serial 16 -bit digital-to-analog converter, $A \mathrm{mp}=$ microwave power amplifier, Iso=microwave isolator.

tential source of frequency bias [3], the entire microwave power control unit is shielded by a metal enclosure. Low frequency signals (power and data) that pass through the bulkheads encounter $9 \mathrm{GHz}$ notch filters to prevent microwave signals from escaping by these paths.

The presence of spurious, discrete sidebands in the spectrum of the microwave radiation can also lead to errors in the determination of the center of the Ramsey peak [4]. To reduce the coupling of external RF sources to the attenuator and microwave amplifier, control signals are brought through the bulkhead of

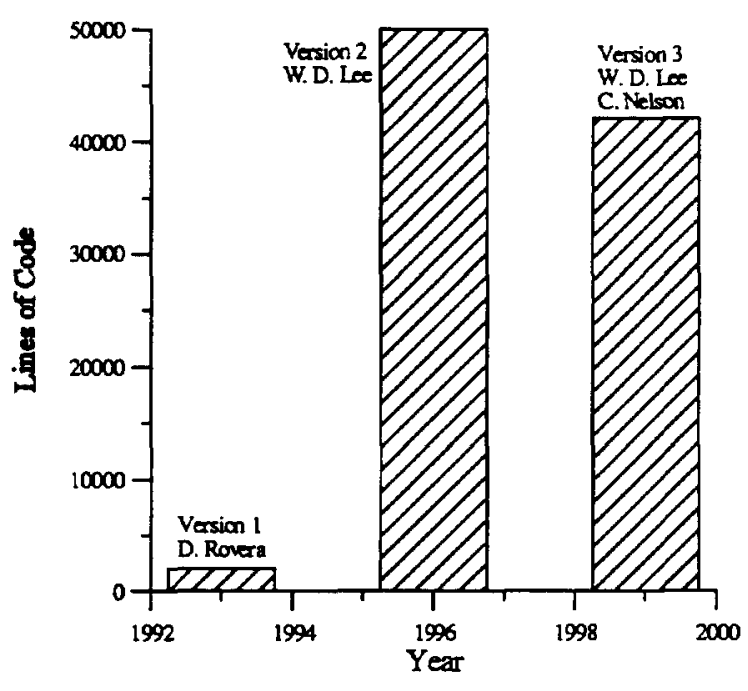

Figure 3: Source Code Size.

the enclosure in digital form. Ground loops are then eliminated using a digital optical isolator. This serial data stream is loaded into a 16-bit digital-to-analog converter which drives the attenuator. The loading of the data into the DAC is synchronized with the digital servo operation to ensure that the DAC has sufficient time to settle and that no data edges are present while the atomic beam fluorescence is measured.

After the attenuator, the power is monitored using a DC-substitution power meter. Absolute accuracy for this measurement is not critical since the meter is calibrated with respect to optimum power for the clock transition. This calibration has been refined using a set of new software tools developed specifcally for CRL-O1. Briefly, optimum power is located by performing a slow square-wave power modulation analogous to using slow square-wave frequency modulation to locate the peak of the Ramsey fringe.

\section{SOFTWARE}

Modern primary frequency standards make extensive use of computers for digital servos, and data acquisition and analysis. Undetected logic or timing errors in software can give rise to unacceptable frequency errors. Thus, software must be testable and maintainable. We have found that traditional procedural programming languages like $\mathrm{C}$ or FORTRAN are not suitable for large, demanding applications like primary frequency standards.

Figure 3 shows the size of the source code we have used for our primary frequency standards. Version 1 of the code, written by $D$. Rovera, consisted of a few modules totaling $\approx 2,000$ lines of $\mathrm{C}$ source code. In 
1993 we began developing additional code to reduce the uncertainty of NIST-7 and to facilitate the evaluation process. After 3 years, the code had grown to $\approx 50,000$ lines. The CRL-O1 project presented us with the opportunity to completely rewrite the operating and analysis software using modern objectoriented programming in order to curb code inflation. $\mathrm{C}++$ was selected as the language. Remarkably, the improvements in software functionality in version 3 have lead to a decrease in the total size of the source code. This is a consequence of reusing source code through the object-oriented mechanism of "software inheritance." Inheritance allows new software modules to contain all the verified, tested functionality of any previously written modules without explicitly copying source code. Older features may be modified or removed, while new features may be added. Inheritance reduces the total amount of source code that must be tested, since only the new or modified features represent additional code. This also makes code maintenance simpler, since a particular algorithm resides in a single source file, propagating through inheritance, not by source code duplication.

Previous versions of the software required the user to manually adjust the servo gain. The new software library includes improvements in the digital frequency servo. The DDS frequency corrections form a time series $\Delta f[n]$, whose mean $\overline{\Delta f}$ represents the raw frequency offset of the reference oscillator from the Ramsey resonance. An estimate of the corresponding statistical uncertainty is

$$
\sigma_{\frac{2}{\Delta f}}=\frac{\sigma_{\Delta f}^{2}}{N}
$$

where

$$
\sigma_{\Delta f}^{2}=\left\langle\Delta f^{2}[n]\right\rangle-\langle\Delta f[n]\rangle^{2},
$$

and $N$ is the length of the time series. Our analysis of the digital servo has shown that (1) is unbiased only when the digital servo's integral gain is made equal to the "ideal gain", the reciprocal of the slope of the frequency error signal at line center [5]. The slope of the error signal is sensitive to many operational parameters including atom flux, detection-laser intensity, and microwave power. The new software library automatically determines the ideal gain (to a specified precision) before the measurement of every time series $\Delta f[n]$. A test of the integral gain adjustment is the Allan deviation at small $\tau$. Figure 4 shows the frequency stability of CRL-O1, relative to a hydrogen maser. For small $\tau$, the points in Figure 4 are in excellent agreement with the white frequency-noise fit, $1.4 \cdot 10^{-12} \tau^{-1 / 2}$. This is crucial in order that the estimate of the statisital uncertainty (1) of the mean frequency difference be unbiased.

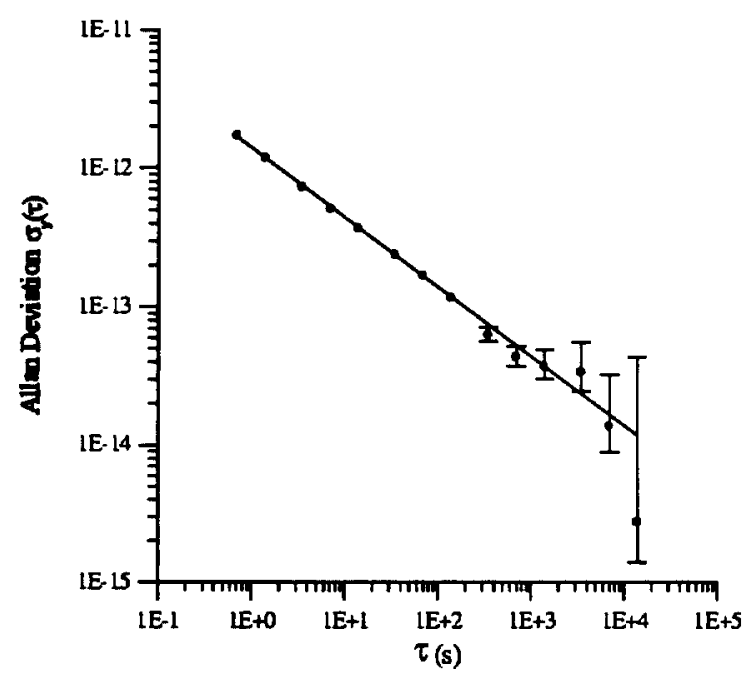

Figure 4: Frequency stability of CRL-O1 relative to a hydrogen maser. The solid line is a fit to the data with $\sigma_{y}(\tau)=1.4 \cdot 10^{-12} \tau^{-1 / 2}$.

This new library of software tools operates not only CRL-O1, but also the NIST cesium fountain, NISTF1, and is currently being adapted to NIST-7. The software library has proved useful in solving many other data acquisition and control problems in time and frequency metrology at NIST.

\section{Accuracy Evaluation}

Accuracy evaluations were performed at NIST in parallel on CRL-O1 and NIST-7 beginning in July 1998. The fractional-frequency difference $y_{\mathrm{CRL}-\mathrm{O} 1}$ relative to a hydrogen maser was determined from a set of 16 frequency measurements, each spanning 12 hours:

$$
y_{\mathrm{CRL}-01}=-8561 \cdot 10^{-15} \pm 5 \cdot 10^{-15} .
$$

The measurements include 5 different $\mathrm{C}$-field values and 2 different microwave powers. The fractionalfrequency difference $y$ NIST -7 relative to the same hydrogen maser was calculated from 12 freqency measurements, each lasting 12 hours:

$$
y_{\text {NIST }-7}=-8560 \cdot 10^{-15} \pm 4 \cdot 10^{-15} \text {. }
$$

Thus the two primary standards are in excellent agreement, well within the statistical uncertainties $(1 \sigma)$ given in (3) and (4).

The frequencies of both standards are charaterized by a Type $B$ standard uncertainty of $10 \cdot 10^{-15}$ as shown in Table 1. Particular values for the secondorder Doppler shift are given for a nominal oven temperature $\left(100^{\circ} \mathrm{C}\right)$ and microwave power $(2.5 \mathrm{~dB}$ below optimum). During a frequency measurement, the 
Table 1: Frequency Biases and Their Type B Standard Uncertainty for NIST $-\bar{i}$ and CRL-O1. Units are fractional frequency $\times 10^{-15}$.

\begin{tabular}{|c|c|c|c|}
\hline $\begin{array}{l}\text { Physical } \\
\text { Effect }\end{array}$ & $\begin{array}{c}\text { NIST-7 } \\
\text { Bias }\end{array}$ & $\begin{array}{c}\text { CRL-O1 } \\
\text { Bias }\end{array}$ & Type B \\
\hline $\begin{array}{l}\text { Second-order } \\
\text { Doppler }\end{array}$ & -293 & -278 & 1 \\
\hline $\begin{array}{l}\text { Second-order } \\
\text { Zeeman }\end{array}$ & $147,609.7$ & $152,592.3$ & 0.1 \\
\hline Cavity pulling & -5 & 0 & 0.5 \\
\hline Rabi pulling & $\leq 0.1$ & $\leq 0.1$ & 0.1 \\
\hline $\begin{array}{l}\text { Cavity phase } \\
\text { (end-to-end) }\end{array}$ & \pm 622 & \pm 225 & 0.7 \\
\hline $\begin{array}{l}\text { Cavity phase } \\
\text { (distributed) }\end{array}$ & 0 & 0 & 4 \\
\hline $\begin{array}{l}\text { Fluorescence } \\
\text { light }\end{array}$ & -0.01 & -0.01 & 9 \\
\hline Blackbody & -20 & -20 & 0.5 \\
\hline Gravitation & +180 & +180 & 0.5 \\
\hline \multicolumn{4}{|c|}{ Electronics } \\
\hline $\begin{array}{l}\text { RF spectral } \\
\text { purity }\end{array}$ & 0 & 0 & 0.1 \\
\hline $\begin{array}{l}\text { Integrator } \\
\text { offset }\end{array}$ & 0 & 1 & 1 \\
\hline $\begin{array}{l}\text { AM on } \mathrm{RF} \text { or } \\
\text { laser }\end{array}$ & -2 & 0 & 1 \\
\hline $\begin{array}{l}\text { Microwave } \\
\text { leakage }\end{array}$ & 0 & 0 & 0.1 \\
\hline \multicolumn{3}{|c|}{ Type B Standard Uncertainty } & 10 \\
\hline
\end{tabular}

digital frequency servo stabilizes the value of the Cfield and thus the second-order Zeeman shift. The distributed cavity phase shift is the result of calculations based upon our theoretical understanding of the system. The gravitational bias uncertainty is based upon the work of $\mathrm{M}$. Weiss. The large uncertainty in fluorescent light shift was assigned because a large. unexplained shift has been seen with an alternative optical pumping arrangement.

\section{CONCLUSION}

CRL and NIST have completed the development of CRL-O1, an improved thermal-atomic-beam primary frequency standard. Improvements in the physics package, electronics, and software have yielded a primary standard that is capable of nearly automated evaluations. Thorough accuracy evaluations of both CRL-OI and NIST-7 were performed at NIST. The results, each with a Type B standard uncertainty of $10 \cdot 10^{-15}$ have been reported to BIPM.

\section{REFERENCES}

[1] R. E. Drullinger, D. J. Glaze, J. P. Lowe, and J. Shirley, "The NIST Optically Pumped Cesium Frequency Standard," IEEE Trans. Instrum. Meas., vol. 40, no. 2, pp. 162-164, April 1991.

[2] J. F. Garcia Nava, F. L. Walls, J. H. Shirley, W. D. Lee, and M. C. Delgado Aramburo, "Environmental Effects in Frequency Synthesizers for Passive Frequency Standards," in Proceedings of the 1996 IEEE International Frequency Control Symposium, pp. 973-979.

[3] W. D. Lee, J. P. Lowe, J. H. Shirley, and R. E. Drullinger, "Microwave Leakage as a Source of Frequency Error and Long Term Instability in Cesium Atomic Beam Frequency Standards," in Proceedings of the 8th European Frequency and Time Forum, pp. 513-516.

[4] W. D. Lee, J. H. Shirley, F. L. Walls, and R. E. Drullinger, "Systematic Errors in Cesium Beam Frequency Standards Introduced by Digital Control of the Microwave Excitation," in Proceedings of the 1995 IEEE International Frequency Control Symposium, pp. 113-117.

[5] W. D. Lee, J. H. Shirley, and R. E. Drullinger, "Analysis of Frequency Biases and Noise in Sampled Digital Frequency Servos for Primary Frequency Standards," in Proceedings of the 1997 IEEE International Frequency Control Symposium, pp. 249-252. 